\title{
INTERDISCIPLINARIDADE E INTERPROFISSIONALIDADE NA ESTRATÉGIA SAÚDE DA FAMÍLIA
}

\author{
INTERDISCIPLINARY AND INTERPROFESSIONALITY IN THE FAMILY HEALTH STRATEGY \\ INTERDICIPLINARIO E INTERPROFESIONALIDAD EN LA ESTRATEGIA SALUD DE LA FAMILIA
}

\author{
Danyelle Nóbrega de Farias ${ }^{1}$ \\ Kátia Suely Queiroz Silva Ribeiro ${ }^{2}$ \\ Ulisses Umbelino dos Anjos ${ }^{3}$ \\ Geraldo Eduardo Guedes de Brito ${ }^{4}$
}

Resumo A interdisciplinaridade e sua correlação prática, a interprofissionalidade, são essenciais ao trabalho na Estratégia Saúde da Família. Objetivou-se caracterizar a perspectiva interdisciplinar na Estratégia Saúde da Família numa capital do Brasil, relacionando-a à interprofissionalidade. Realizou-se estudo transversal, exploratório e descritivo, com abordagens qualitativa e quantitativa. Os dados foram coletados no período de junho a novembro de 2014 por meio de questionários e observação participante. Os resultados do questionário foram convertidos em escores, permitindo classificar o nível de interdisciplinaridade. Os achados da observação, registrados no diário de campo, foram submetidos à análise de conteúdo e discutidos em paralelo com os achados quantitativos. Constatou-se, nos questionários, que a dimensão 'acolhimento' foi a única que apresentou média disponibilidade para práticas que poderiam viabilizar a interdisciplinaridade. Os resultados apontaram que a percepção dos profissionais de saúde sobre interdisciplinaridade foi positiva, mas verificaram-se contradições desses achados com o observado in locus. Na observação participante, constatou-se que os profissionais precisam trilhar alguns caminhos no sentido de materializar a interdisciplinaridade em práticas interprofissionais colaborativas. Destaca-se a necessidade de ações em nível da gestão que favoreçam essas práticas; a educação permanente como estratégia de enfrentamento das dificuldades de integração; e o investimento subjetivo dos trabalhadores na mesma direção.

Palavras-chave comunicação interdisciplinar; pesquisa interdisciplinar; equipe de saúde interdisciplinar; relações interprofissionais.
Abstract Interdisciplinarity and its practical correlation, interprofessionality, are essential to the work in the Family Health Strategy. The objective was to characterize the interdisciplinary perspective of Family Health Strategy workers in a Brazilian capital, linking it to interprofessionality. Transversal, exploratory and descriptive study, developed from the qualitative and quantitative approaches. Data were collected from june to november 2014 through questionnaires and participant observation. The results of the questionnaire were converted into scores, allowing to classify the level of interdisciplinarity. The observation findings, recorded in the field diary, were submitted to content analysis and discussed in parallel with the quantitative findings. It was verified, in the questionnaires, that the dimension 'reception' was the only one that presented medium availability for practices that can make interdisciplinarity feasible. The results indicate that the health professionals' perception about interdisciplinarity was positive, however, contradictions of these findings with the ones observed locus. In the participant observation, it was verified that the professionals need to follow some paths in the sense of materializing the interdisciplinarity in collaborative interprofessional practices. It is important to emphasize the need for management-level actions that favor these practices; Permanent education as a strategy for coping with integration difficulties; And the subjective investment of workers in the same direction.

Keywords interdisciplinary communication; interdisciplinary research; team of interdisciplinary health; interprofessional relations. 


\section{Introdução}

São inegáveis os avanços conquistados pela implantação do Sistema Único de Saúde (SUS) ao longo das últimas décadas. No entanto, há evidências de que o setor de saúde brasileiro encontra-se em um cenário de crise no que se refere à sua forma de cuidar, devido a práticas ainda baseadas predominantemente na abordagem biologicista (Malta e Merhy, 2010). Tal panorama representa uma importante fragilidade para consolidar a mudança do modelo assistencial almejada pelos ideários da Reforma Sanitária Brasileira.

No Brasil, atenção primária à saúde (APS), considerada o principal mecanismo de reorganização do sistema de saúde, é preferencialmente representada pela Estratégia Saúde da Família (ESF), à qual se atribui o desafio de romper com a lógica tradicional de assistência à saúde para que o cuidado seja baseado na família e no contexto social, por meio de uma atenção com base interdisciplinar (Costa et al., 2009) e atuação interprofissional.

Para Feuerwerker e Cecílio (2007), a construção histórica do trabalho em saúde foi fortemente influenciada pelo expressivo avanço tecnológico e pela especialização, com consequente desenvolvimento e aprimoramento da capacidade diagnóstica e de tratamento dos problemas de saúde. A consequência desse reducionismo e da estratificação dos indivíduos é materializada na dificuldade de construir e ressignificar os conhecimentos, por meio dos quais se possam fazer intervenções individuais e coletivas, baseadas no conceito de determinação social do processo saúde-doença, que impactem positivamente as condições de vida e de saúde da população, quebrando com a fragmentação do saber da saúde.

Os conhecimentos descontextualizados e as disciplinas abordadas de forma segmentada na formação dos trabalhadores do setor saúde comprometem o alcance de uma visão ampliada do processo saúde-doença e a capacidade de trabalhar em equipe, o que implica na baixa resolutividade do cuidado. Evidencia-se, assim, a necessidade de uma abordagem interdisciplinar e uma intervenção interprofissional das questões de saúde. Japiassu (1976) assinala que a interdisciplinaridade compreende a intensidade das trocas entre os especialistas, a integração e a articulação de diferentes saberes e práticas que geram uma intervenção, uma ação comum, que horizontaliza saberes e relações de poder.

No contexto atual do setor saúde há um debate crescente acerca do trabalho interprofissional e da necessidade de se fazer uma distinção entre interdisciplinaridade e interprofissionalidade. Diferentemente da perspectiva apresentada por Japiassu, aqui mencionada, tal debate sugere que o termo interdisciplinaridade refere-se à integração de saberes, e interprofissionalidade à integração de práticas (Furtado, 2009), mediante a articulação intencional e colaborativa entre distintas profissões (Costa et al., 2015). 
O trabalho interprofissional em saúde, por meio da prática colaborativa, é apresentado como uma das melhores formas de se enfrentarem os desafios altamente complexos do setor saúde e a concretização da interdisciplinaridade. Enquanto esta última diz respeito à esfera das disciplinas, ciências ou áreas de conhecimento, a interprofissionalidade corresponde à prática profissional em que se desenvolve o trabalho em equipe de saúde, articulando diferentes campos de práticas e fortalecendo a centralidade no usuário e suas necessidades na dinâmica da produção dos serviços de saúde (Peduzzi et al., 2013). Trabalhar em equipe interprofissional significa atuar com profissionais de diversas formações na saúde, dispostos a transitar entre as áreas específicas de formação e de promover, além do ensino, a atuação interprofissional (Barr et al., 2005; World Health Organization, 2010).

Salienta-se que a interdisciplinaridade tem grande potencial para dar sustentação a ações integrais e mais resolutivas, sobretudo quando centradas nas necessidades do usuário. Nesse contexto, o estudo aqui apresentado objetivou caracterizar a abordagem interdisciplinar de trabalhadores de nível superior da Estratégia Saúde da Família em uma capital do Brasil, relacionando-a à prática interprofissional e colaborativa.

\section{Desenho do estudo}

O estudo que deu origem a este artigo foi do tipo transversal e descritivo de abordagem qualiquantitativa, desenvolvido com trabalhadores de unidades de saúde da família (USFs) dos cinco distritos sanitários (DSs) do município de João Pessoa, capital da Paraíba.

A população-alvo do componente quantitativo do estudo foi composta de 522 trabalhadores de nível superior (175 cirurgiões-dentistas, 179 enfermeiros e 168 médicos) da ESF da área urbana de João Pessoa. A amostra mínima $(n=261)$ foi calculada de acordo com a fórmula proposta por Bussab, Miazaki e Andrade (1990), em que se adota a estratificação proporcional ao número total de cada categoria profissional de interesse (cirurgiões-dentistas $=87$; enfermeiros $=89$; médicos $=85$ ). Foi estimada uma perda de $40 \%$ da amostra por instrumentos não devolvidos aos pesquisadores, com base nos resultados de Zanetti e colaboradores (2010). Assim, foram distribuídos 365 questionários.

Para coletar os dados quantitativos, foi elaborado um questionário autoaplicável com base nos referenciais teóricos de Japiassu (1976) e Saupe e colaboradores (2005), submetido à análise crítica de quatro especialistas no tema e a um estudo-piloto. A versão final do instrumento continha 27 assertivas relacionadas à interdisciplinaridade, divididas em sete dimensões: acolhimento (duas questões), comunicação (três questões), atitude (duas questões), atividades coletivas (dez questões), disponibilidade de tempo (duas questões), planejamento (quatro questões) e atividades individuais (quatro questões). 
Para Furtado (2009), a interprofissionalidade é o correlato da interdisciplinaridade nas práticas de saúde. Nesse sentido, as dimensões referentes à integração das práticas profissionais dos resultados do estudo foram analisadas como interprofissionalidade, e aquelas relacionadas à integração dos saberes, como interdisciplinaridade.

A amostra foi selecionada mediante o sorteio das equipes de saúde por distrito sanitário, através do cadastro das equipes de saúde da família disponibilizado pela Secretaria Municipal de Saúde, o que se caracteriza como uma escolha por conglomerados. Uma equipe constituída por oito pesquisadores devidamente treinada explicava os objetivos do estudo, convidava os trabalhadores para participar, coletava a assinatura do termo de consentimento livre e esclarecido e, posteriormente, recolhia o questionário.

Os sujeitos foram orientados pela equipe de coleta de dados para assinalar o grau de concordância em cada assertiva das questões, que variava em uma escala graduada de zero (discordo totalmente) a dez (concordo totalmente). Para a apresentação dos resultados, o valor obtido em cada dimensão foi calculado pelo escore médio.

Os escores obtidos em cada questão foram categorizados em três intervalos. Quando o valor obtido encontrava-se no intervalo [0,00; 3,33], foi classificado como 'fraca interdisciplinaridade'; no intervalo $[3,33 ; 6,66]$, como 'média interdisciplinaridade'; e entre $[6,66 ; 10,00]$, como 'forte interdisciplinaridade'.

Quanto ao componente qualitativo, procedeu-se à observação participante, durante dois meses, em uma unidade integrada de saúde da família, com o objetivo de confrontar os resultados quantitativos com a prática cotidiana dos trabalhadores da ESF. Elegeu-se, mediante sorteio, uma unidade integrada de saúde da família, em cujo espaço físico se reuniam quatro equipes de saúde da família, identificadas por nomes de cores (Rosa, Verde, Azul e Laranja), com o intuito de facilitar o registro das atividades acompanhadas.

Para compilar os dados da observação participante, foi utilizado um diário de campo, construído com base nos tópicos definidos de acordo com a conceituação de Japiassu (1976) e de Saupe e colaboradores (2005) para a interdisciplinaridade e que nortearam a investigação in locus: objetivo em comum, linguagem, comunicação, diálogo durante as atividades e o relacionamento da equipe (divisão de tarefas, responsabilidades); momentos em que a interdisciplinaridade foi vivenciada no dia a dia dos profissionais (atendimentos, grupos, reuniões); movimentos em prol da construção da interdisciplinaridade e as dificuldades enfrentadas para essa realização.

Nos turnos de observação, foram acompanhadas as atividades feitas pelos profissionais nas visitas domiciliares, os atendimentos em consultório e as atividades coletivas (escuta qualificada, atividades educativas, reuniões de equipe e campanhas). No tocante às atividades educativas, acompanharam-se as ações realizadas com diferentes públicos, abordagens e finalidades, desen- 
volvidas no espaço da USF e no território (acompanhamento de hipertensos e diabéticos, atividades de sala de espera e ação educativa para prevenção do câncer de mama).

Os dados foram analisados qualitativa e quantitativamente. No primeiro caso, por meio da análise temática segundo Bardin (2011). Para isso, realizou-se uma leitura flutuante do material escrito, constituído dos registros do diário de campo. Posteriormente, fez-se uma segunda leitura, seguindo-se as fases de pré-análise, codificação, tratamento dos resultados e interpretação. As categorias de análise foram definidas a priori com base nos tópicos que orientaram a elaboração do diário de campo. Dessa maneira, foi possível discutir à luz da literatura e comparar com os achados dos questionários.

Para a análise quantitativa, o controle de qualidade e o processamento dos dados, recorreu-se ao programa Epi Info ${ }^{\mathrm{TM}}$. Para caracterizar a amostra, calcularam-se as prevalências quanto às respostas relacionadas ao trabalho interdisciplinar na ESF. Foi testada a normalidade ante as variáveis - a distribuição resultante não era normal. Assim, utilizou-se o teste de Kruskal-Wallis para verificar a diferença entre os grupos e posteriormente Mann-Whitney entre os pares. O teste de Mann-Whitney foi eleito para verificar a heterogeneidade das respostas, considerando-se o nível de significância 5\%.

A pesquisa ${ }^{5}$ apresentada neste artigo foi autorizada pela Secretaria Municipal de Saúde de João Pessoa, submetida à apreciação e aprovada, de acordo com o protocolo n. 25443413.2.0000.5188, pelo Comitê de Ética em Pesquisa do Centro de Ciências da Saúde da Universidade Federal da Paraíba, e submetida à Plataforma Brasil, aprovada com o protocolo n. 0030/14, respeitando a resolução n. 466/12. Na coleta dos dados, solicitou-se aos participantes a assinatura do termo de consentimento livre e esclarecido para inclusão na pesquisa, que contou com o apoio financeiro da chamada universal MCTI/CNPq n. 14/2014.

\section{A interdisciplinaridade e a interprofissionalidade na Estratégia Saúde da Família: entre o discurso e a prática}

Foram entrevistados 342 trabalhadores: enfermeiros $(n=127)$, médicos $(n=97)$ e cirurgiões-dentistas $(n=118)$ da ESF. A amostra apresentou-se composta predominantemente pelo sexo feminino $(83,3 \%)$ e com média de idade de 45,8 anos $(\mathrm{d} p=13,6)$, concentrados na faixa etária de quarenta anos ou mais $(62,3 \%)$. Essas características podem ser observadas na Tabela 1.

Algumas características de formação dos sujeitos do estudo também foram investigadas. A maioria dos entrevistados declarou ter concluído há mais de 21 anos a graduação da profissão que exercia na saúde da família. Entre os médicos, apenas 13,4\% tinham entre cinco e vinte anos de formação, com expressivos $37,1 \%$ com até quatro anos de graduação, um percentual bem mais elevado do que os $12,7 \%$ dos cirurgiões-dentistas e os 7,9\% dos enfer- 
Tabela 1

\begin{tabular}{|c|c|c|c|c|c|c|c|c|}
\hline \multicolumn{9}{|c|}{ Características gerais da amostra estratificadas por categoria profissional } \\
\hline \multirow{2}{*}{ CARACTERÍSTICA } & \multicolumn{2}{|c|}{ Amostra } & \multicolumn{2}{|c|}{ Enfermeiros } & \multicolumn{2}{|c|}{ Médicos } & \multicolumn{2}{|c|}{ Cirurgiões-dentistas } \\
\hline & $\mathbf{N}$ & $\%$ & $\mathbf{N}$ & $\%$ & $\mathbf{N}$ & $\%$ & $\mathbf{N}$ & $\%$ \\
\hline \multicolumn{9}{|l|}{ Sexo } \\
\hline Feminino & 285 & 83,3 & 125 & 98,4 & 63 & 64,9 & 97 & 82,2 \\
\hline Masculino & 57 & 16,7 & 2 & 1,6 & 34 & 35,1 & 21 & 17,8 \\
\hline Faixa etária & $\mathrm{N}$ & $\%$ & $\mathrm{~N}$ & $\%$ & $\mathrm{~N}$ & $\%$ & $\mathrm{~N}$ & $\%$ \\
\hline Até 29 anos & 54 & 15,8 & 11 & 8,7 & 26 & 26,8 & 17 & 14,4 \\
\hline 30 a 39 anos & 75 & 21,9 & 34 & 26,8 & 18 & 18,6 & 23 & 19,5 \\
\hline 40 a 49 anos & 65 & 19,0 & 29 & 22,8 & 8 & 8,2 & 28 & 23,7 \\
\hline 50 a 59 anos & 85 & 24,9 & 39 & 30,7 & 13 & 13,4 & 33 & 28,0 \\
\hline 60 anos ou mais & 63 & 18,4 & 14 & 11,0 & 32 & 33,0 & 17 & 14,4 \\
\hline
\end{tabular}

meiros. O tempo de formação sugere ser um fator relevante pelo fato de que esses profissionais podem ter enfrentado dificuldade para atuar na perspectiva interdisciplinar do cuidado integral, já que o movimento de mudanças na formação vem sendo mais forte nos últimos dez anos (Machado et al., 2007).

Em relação ao curso de pós-graduação lato sensu, apenas $22,5 \%$ da amostra não havia cursado uma pós-graduação - 92,1\% dos enfermeiros e 80,5\% dos cirurgiões-dentistas têm o título. Quanto à categoria médica, 54,6\% cursaram uma especialização, o que justifica o número expressivo $(37,1 \%)$ de médicos recém-formados na ESF (0-4 anos). Tais resultados, associados a lacunas de formação na graduação, podem representar uma fragilidade de formação/capacitação que compromete a efetivação do trabalho interprofissional.

Quanto mais capacitados forem os profissionais que atuam na ESF, melhores serão os serviços ofertados. Portanto, os cursos de pós-graduação - principalmente os que abordem temas relacionados à saúde coletiva, à interdisciplinaridade e à atuação interprofissional - são cruciais para o desempenho dos profissionais que atuam no SUS e contribuem para melhorar a assistência prestada aos usuários (Batista e Gonçalves, 2011).

$\mathrm{Na}$ análise do questionário, a dimensão 'acolhimento' obteve a média mais baixa $(6,1)$; logo, foi classificada como 'média prática interdisciplinar'. As dimensões 'atividades coletivas' $(7,4)$, 'disponibilidade de tempo' $(7,8)$, 'planejamento' $(7,9)$, 'atividades individuais' $(8,3)$, 'comunicação' $(8,6)$ e 'atitude' $(9,6)$ tiveram suas práticas classificadas como 'forte interdisciplinaridade'. Nenhuma dimensão foi classificada como 'fraca interdisciplinaridade'. A análise desses resultados, isoladamente, indica que a percepção dos sujeitos do estudo quanto à integração no nível dos saberes (interdisciplinar) e das práticas (interprofissional) na Estratégia Saúde da Família foi 
positiva, uma vez que mais de $90 \%$ das variáveis investigadas apresentaram médias superiores a sete, e estatisticamente os grupos não apresentaram diferenças entre si.

No Quadro 1, constam os resultados relativos às práticas das equipes de saúde da família verificados durante a observação participante. As informações coletadas foram categorizadas de acordo com a literatura e apresentadas

\section{Quadro 1}

\begin{tabular}{|c|c|c|c|c|c|c|c|}
\hline \multicolumn{8}{|c|}{ Caracterização das ações realizadas com base na observação participante das equipes de saúde da família } \\
\hline & & $\begin{array}{c}\text { Visita } \\
\text { domiciliar }\end{array}$ & Acolhimento & Atendimento & $\begin{array}{l}\text { Atividades } \\
\text { educativas }\end{array}$ & Reuniões & Campanhas \\
\hline \multirow{4}{*}{ 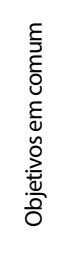 } & Rosa & - & - & $\begin{array}{l}\text { Centrado em } \\
\text { uma pessoa }\end{array}$ & $\begin{array}{l}\text { Centrado em } \\
\text { uma pessoa }\end{array}$ & Parcialmente & Totalmente \\
\hline & Verde & - & Totalmente & - & Parcialmente & Totalmente & Totalmente \\
\hline & Azul & $\begin{array}{l}\text { Centrado em } \\
\text { uma pessoa }\end{array}$ & - & - & Totalmente & Totalmente & Totalmente \\
\hline & Laranja & - & Parcialmente & Parcialmente & Parcialmente & Parcialmente & Totalmente \\
\hline \multirow{6}{*}{ 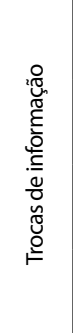 } & Rosa & - & - & $\begin{array}{l}\text { Nenhuma } \\
\text { frequência }\end{array}$ & $\begin{array}{c}\text { Pouca } \\
\text { frequência }\end{array}$ & $\begin{array}{c}\text { Pouca } \\
\text { frequência }\end{array}$ & $\begin{array}{c}\text { Pouca } \\
\text { frequência }\end{array}$ \\
\hline & Verde & - & Pouca frequência & - & $\begin{array}{c}\text { Média } \\
\text { frequência }\end{array}$ & $\begin{array}{c}\text { Muita } \\
\text { frequência }\end{array}$ & $\begin{array}{c}\text { Muita } \\
\text { frequência }\end{array}$ \\
\hline & $\Delta>$ T & Nenhuma & & & Muita & Muita & Muita \\
\hline & & frequência & & & frequência & frequência & frequência \\
\hline & & & Nenhuma & Pouca & Pouca & Média & Média \\
\hline & Laranija & 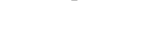 & frequência & frequência & frequência & frequência & frequência \\
\hline \multirow{4}{*}{$\frac{\circ}{\frac{0}{0}}$} & Rosa & - & - & Vertical & Vertical & Vertical & Vertical \\
\hline & Verde & - & Horizontal & - & Horizontal & Horizontal & Horizontal \\
\hline & Azul & Horizontal & - & - & Horizontal & Horizontal & Horizontal \\
\hline & Laranja & - & Vertical & Vertical & Horizontal & Horizontal & Horizontal \\
\hline \multirow{4}{*}{ 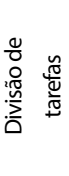 } & Rosa & - & - & Não & Não & Sim & Sim \\
\hline & Verde & - & Sim & - & Sim & Sim & Sim \\
\hline & Azul & & - & - & Sim & Sim & Sim \\
\hline & Laranja & - & Não & Não & Sim & Sim & Sim \\
\hline \multirow{4}{*}{ 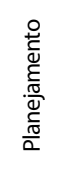 } & Rosa & - & - & Sim & Não & Sim & Sim \\
\hline & Verde & - & Não & - & Sim & Sim & Sim \\
\hline & Azul & Sim & - & - & Sim & Sim & Sim \\
\hline & Laranja & - & Não & Sim & Sim & Sim & Sim \\
\hline \multirow{4}{*}{ 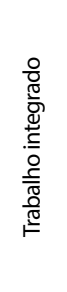 } & Rosa & - & - & Desintegrado & Desintegrado & $\begin{array}{c}\text { Parcialmente } \\
\text { integrado }\end{array}$ & $\begin{array}{c}\text { Parcialmente } \\
\text { integrado }\end{array}$ \\
\hline & Verde & - & $\begin{array}{c}\text { Parcialmente } \\
\text { integrado }\end{array}$ & - & $\begin{array}{c}\text { Parcialmente } \\
\text { integrado }\end{array}$ & Integrado & $\begin{array}{c}\text { Parcialmente } \\
\text { integrado }\end{array}$ \\
\hline & Azul & Desintegrado & - & - & Integrado & Integrado & Integrado \\
\hline & Laranja & - & Desintegrado & $\begin{array}{c}\text { Parcialmente } \\
\text { integrado }\end{array}$ & $\begin{array}{c}\text { Parcialmente } \\
\text { integrado }\end{array}$ & Integrado & $\begin{array}{c}\text { Parcialmente } \\
\text { integrado }\end{array}$ \\
\hline
\end{tabular}

Fonte: Os autores (Diário de campo, 2014). 
como síntese dos achados, tendo em vista as atividades acompanhadas versus os tópicos importantes para caracterizar o trabalho interdisciplinar.

O estudo apontou que há disponibilidade para a troca de conhecimentos de modo a viabilizar a interdisciplinaridade. Todavia, os enfermeiros, os médicos e os cirurgiões-dentistas ainda precisam trilhar alguns caminhos nesse processo para compreender os conceitos e aplicá-los, o que foi evidenciado pelas respostas ao questionário não uniformes sobre as dimensões investigadas na pesquisa e, por diversas vezes, em desacordo com o que foi vivenciado na prática in locus, conforme pôde ser observado na comparação entre as médias nas dimensões e o Quadro 1.

$\mathrm{Na}$ análise da dimensão 'acolhimento', ficou demonstrado que os profissionais estudados referiram que não há integração entre eles durante a escuta qualificada (Tabela 2). Durante a observação participante, verificou-se que essa atividade era centralizada na figura de um profissional e desconexa do objetivo de estabelecer um vínculo entre o trabalhador e o usuário, a universalidade do acesso e a classificação de risco, conforme preconizado pela Política Nacional de Humanização (Brasil, 2010).

A prática do acolhimento tem sido estimulada nos serviços de saúde, com o propósito de racionalizar o processo de atendimento e melhorar a resolutividade, porém é uma mudança que requer a integração de todos os profissionais. Além disso, por meio da observação participante, pode-se perceber que o acolhimento ainda não é compreendido de forma unificada pelos profissionais de saúde, a exemplo do que foi evidenciado no estudo de Garuzi e colaboradores (2014) e assinalado como fragilidade no serviço.

O acolhimento propõe uma reorganização dos serviços de saúde e das relações entre trabalhadores e usuários, de forma que se desloque o eixo centrado no médico para uma equipe multiprofissional - que se encarrega da escuta do usuário, da resolução do problema de saúde do usuário, de qualificar a relação trabalhador-usuário. Essa atividade deve ser conduzida por parâmetros humanitários, de solidariedade e cidadania, de modo a viabilizar um serviço de saúde organizado e com o compromisso de cuidado. Contudo, a pesquisa mostrou que os entrevistados sentiam dificuldade de compreender o conceito e sua prática. Clementino e colaboradores (2015) apontam que o acolhimento é uma importante diretriz, por meio da qual os usuários podem ter mais acesso aos serviços de saúde. Assim, sua execução facilita a entrada no serviço, reduz as barreiras quanto ao tempo de espera e ao mau direcionamento de consultas e facilita a eleição de prioridades e o aumento do vínculo.

$\mathrm{Na}$ avaliação da dimensão referente às 'atividades coletivas', incluem-se questões relativas ao desenvolvimento dessas atividades na perspectiva de promover a interação entre os profissionais, a pactuação de conceitos, os métodos e os objetivos, a hierarquia e a corresponsabilidade em atividades como grupos, reuniões e campanhas. Embora as respostas dos questionários (Tabela 2) 
Tabela 2

\begin{tabular}{|c|c|c|c|c|}
\hline Dimensão & Amostra & Enfermeiros & Médicos & Cirurgiōes-dentistas \\
\hline Acolhimento & 6,11 & 5,55 & 6,60 & 5,86 \\
\hline $\begin{array}{l}\text { No momento do acolhimento, a escuta } \\
\text { qualificada é realizada juntamente com } \\
\text { outros profissionais. }\end{array}$ & 6,13 & 6,02 & 6,60 & 5,86 \\
\hline $\begin{array}{l}\text { Considero necessário que todos os profissionais } \\
\text { da equipe contribuam para sua execução. }\end{array}$ & 6,10 & 5,09 & 6,60 & 5,86 \\
\hline Atividades coletivas & 7,21 & 7,41 & 7,09 & 7,10 \\
\hline $\begin{array}{l}\text { Costumo desenvolver atividades em conjunto } \\
\text { com estudantes/profissionais de outros } \\
\text { núcleos profissionais. }\end{array}$ & 7,67 & 8,20 & 7,68 & 7,10 \\
\hline $\begin{array}{l}\text { Na organização de grupos operativos ou } \\
\text { campanhas, existe um acordo prévio quanto aos } \\
\text { conceitos e à metodologia a serem utilizados, } \\
\text { tendo como base o objetivo do trabalho. }\end{array}$ & 8,53 & 8,80 & 8,23 & 8,48 \\
\hline $\begin{array}{l}\text { Na realização de grupos operativos, cada } \\
\text { profissional participa expondo os conhecimentos } \\
\text { relativos ao seu núcleo profissional, havendo } \\
\text { interseção entre os saberes. }\end{array}$ & 2,89 & 2,73 & 2,65 & 3,27 \\
\hline $\begin{array}{l}\text { Respeito opiniões e sugestões dos outros } \\
\text { profissionais. }\end{array}$ & 9,49 & 9,46 & 9,45 & 9,56 \\
\hline $\begin{array}{l}\text { Não existe uma hierarquia quanto às } \\
\text { responsabilidades estabelecidas. }\end{array}$ & 1,82 & 1,54 & 2,21 & 1,81 \\
\hline $\begin{array}{l}\text { O cronograma das atividades de hiperdia, } \\
\text { pré-natal e puericultura é cumprido por todos } \\
\text { os profissionais de minha equipe. }\end{array}$ & 8,08 & 8,00 & 8,37 & 7,94 \\
\hline $\begin{array}{l}\text { Em dias de hiperdia, pré-natal e puericultura, } \\
\text { costumo interagir com os colegas da equipe } \\
\text { sobre alguns casos atendidos na unidade de } \\
\text { saúde da família. }\end{array}$ & 8,25 & 8,66 & 8,32 & 7,75 \\
\hline Em uma visita domiciliar, após identificar a & & & & \\
\hline $\begin{array}{l}\text { necessidade de encaminhamento, o caso é } \\
\text { compartilhado com outros profissionais. }\end{array}$ & 9,05 & 9,46 & 8,75 & 8,86 \\
\hline $\begin{array}{l}\text { Realizo consultas compartilhadas (com outros } \\
\text { profissionais). }\end{array}$ & 7,98 & 8,67 & 7,28 & 7,82 \\
\hline $\begin{array}{l}\text { No caso em que as visitas domiciliares são } \\
\text { realizadas com atendimento compartilhado, } \\
\text { existe discussão/troca de experiências entre os } \\
\text { núcleos profissionais que se encontram na visita. }\end{array}$ & 8,40 & 8,67 & 8,01 & 8,43 \\
\hline
\end{tabular}

Fonte: Os autores.

indiquem a realização de grupos operativos com participação de todos os profissionais, constatou-se na observação que essa participação ficava restrita à exposição de conhecimentos relativos ao seu núcleo profissional, sem que houvesse interseção entre os saberes - o que se configurou como uma 
limitação dessa dimensão. Para que a prática profissional seja mais efetiva, é necessário que haja interdisciplinaridade, ou seja, que a interação dos conhecimentos mostre a interdependência entre os saberes e as necessidades de articulação entre as áreas da saúde que atuem num mesmo ambiente de trabalho (Brasil, 2009).

A observação participante permitiu identificar que existia certa hierarquia nas responsabilidades estabelecidas dentro do local de trabalho. Com base nos registros do diário de campo, viu-se na figura do gerente da ESF uma relação de comunicação vertical, o que se agravava pelo fato de a figura gerencial ser recebida pelos profissionais como um 'vigia/coordenador'. Porém, não se observou nenhuma situação que mostrasse essa hierarquização com relação às decisões. Estudos recentes sobre o trabalho em equipe na ESF (Oliveira, Pires e Parente, 2011; Pires e Göttems, 2010) revelaram falta de responsabilidade coletiva do trabalho e baixo grau de interação entre as categorias profissionais. Apesar do discurso de teor igualitário, os membros das equipes de saúde da família mantêm representações sobre hierarquia entre profissionais (médico, enfermeiro e cirurgião-dentista) e usuários, nível superior e nível médio de educação. Segundo Oliveira, Pires e Parente (2011), embora os profissionais não reconheçam, as relações de poder vão se tecendo no interior das equipes, numa forma de polarizar que se estabelece entre as pessoas e divide as que 'podem' mais das que podem menos, estabelecendo uma hierarquia nas relações de forma paradoxal com o previsto nas diretrizes que orientam o trabalho na Estratégia Saúde da Família, o qual deve ser interdisciplinar e interprofissional (Brasil, 2012).

Na organização de grupos operativos ou de campanhas, constatou-se com base no diário de campo a existência de um acordo prévio sobre os conceitos e a metodologia que seriam utilizados, tendo como base o objetivo do trabalho, em que se procurava respeitar as opiniões e as sugestões dos outros profissionais. Nos espaços das reuniões de equipe, os profissionais conseguiam participar das discussões juntamente com os colegas de trabalho, e durante o planejamento das atividades do serviço havia troca de conhecimentos com profissionais de outros núcleos. Esse fato contribuía para que a interprofissionalidade fosse vivenciada na equipe de saúde da família. Em certa observação de reunião de equipe, presenciou-se uma fala cuja importância é válida destacar, uma vez que enfatiza o valor de todos os atores que compõem a equipe de saúde da família e sua colaboração:

Todos temos problemas (...) o importante é saber respeitar a opinião do outro, solicitar auxílio quando necessário e trabalhar em prol de um objetivo comum (Médico 3).

A construção e a execução de atividades com grupos operativos podem propiciar a prática colaborativa, desde que haja condições objetivas e subjetivas 
para que ela ocorra. A prática colaborativa é compreendida como componente da organização dos serviços e ocorre quando profissionais de saúde de diferentes áreas prestam serviços com base na integralidade da saúde, envolvendo os usuários e suas famílias, cuidadores e comunidades. Exige que tais profissionais estejam dispostos a transitar entre as áreas específicas de formação e articulem seu saber específico com o dos outros, de modo a possibilitar o compartilhamento das ações e delegar atividades, o que aumentaria a resolubilidade dos serviços e a qualidade da atenção à saúde (World Health Organization, 2010; Peduzzi et al., 2013).

Quanto à 'disponibilidade de tempo' (Tabela 3), de forma homogênea, os profissionais estudados relataram ter carga horária para planejar e realizar com a equipe as atividades de grupo, como o acompanhamento de hipertensos e diabéticos, o pré-natal e a puericultura, que eram cumpridas por todos da equipe (médicos, enfermeiros e cirurgiões-dentistas). A dimensão 'tempo' não foi avaliada como uma dificuldade para o trabalho interprofissional. Em contrapartida, na observação participante, pôde-se perceber o fator tempo como limitante para a integração que a interprofissionalidade requer, porquanto os profissionais tendiam a se ausentar de suas atividades laborais antes de cumprir a carga horária e muitas vezes por apresentarem dias de folga alternados, o que dificultava o planejamento e as ações de cunho coletivo.

A dimensão 'planejamento' (Tabela 3) envolveu questões sobre a possibilidade de se discutirem as atividades do serviço de maneira horizontalizada, com troca de conhecimentos e diálogo entre os profissionais da equipe. Os profissionais concebiam a realização do projeto terapêutico singular (PTS), enquanto estratégia do planejamento, como uma fragilidade para o trabalho interprofissional. Essa atividade é sobremaneira importante para o compartilhamento de casos clínicos, o que envolve a presença de todos os profissionais e é realizada quando se torna necessário compreender de forma multidimensional a situação, caracterizando assim a colaboração interprofissional.

Ao se avaliar isoladamente a assertiva, notaram-se médias significativamente baixas das respostas obtidas, o que implica dizer que as três classes profissionais avaliadas pouco realizavam ou não realizavam o PTS. No entanto, em um quesito posterior, os mesmos profissionais deram notas altas à afirmativa que dizia que, ao identificar um caso complexo, eles utilizam o momento da reunião com a equipe de saúde da família para obter informações que ajudem a compreender melhor a situação a fim de traçar um PTS, discordando do que havia sido dito antes. Essas inversões de respostas podem se justificar devido ao longo questionário aplicado aos profissionais que, muitas vezes, dispersos pela ansiedade de responder rapidamente às questões, perdem a atenção e lançam falsas respostas, conforme descrito por De Britto Júnior e Feres Júnior (2011). Essa explicação pode ter gerado escores altos para a dimensão 'planejamento'. 
Tabela 3

Escore das dimensões disponibilidade de tempo, planejamento e atividades individuais estratificadas por categoria profissional

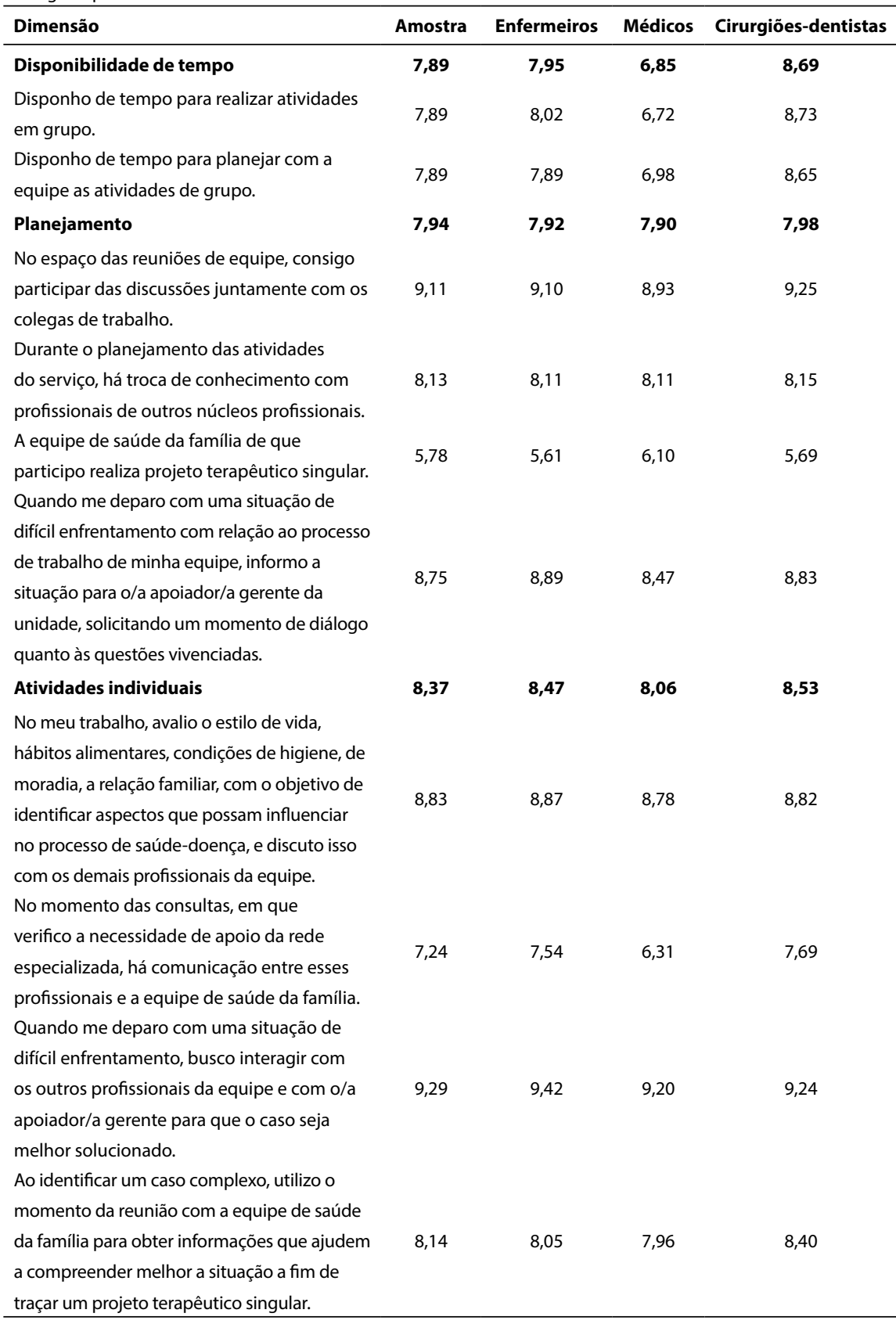

Fonte: Os autores.

Diferentemente da análise quantitativa, durante a observação participante somente uma equipe reiterou o conceito do planejamento para as ações interprofissionais, quando, em suas ações, todos trabalhavam juntos desde a 
ideia até a execução de qualquer atividade. A fala de uma cirurgiã-dentista em reunião de equipe ilustrou bem essa postura:

Trabalhar em ESF não é apenas estar junto fisicamente, é mesmo estando longe ter conversado e programado em equipe; é compartilhar estratégias (Cirurgiã-dentista 3).

A dimensão 'planejamento' relaciona-se com as demais dimensões, uma vez que, para qualquer ação, é necessário programar os caminhos a seguir, saber ouvir, saber falar, planejar, avaliar e trabalhar em conjunto.

A observação participante permitiu verificar que, a despeito dos resultados positivos dos questionários, havia dificuldades em relação à disponibilidade de tempo para atuação colaborativa, ao planejamento conjunto e à realização do PTS - o que se refletia em limitações para o trabalho interprofissional e, consequentemente, comprometia a qualidade da atenção prestada.

Quanto à dimensão 'atividades individuais', o estudo quantitativo (Tabela 3) indica que os problemas de saúde não podem ser entendidos isoladamente, que é preciso haver colaboração e integração de todos os envolvidos no serviço. Os profissionais em questão afirmaram, de forma homogênea, que havia integração de saberes e de práticas nessas atividades. Relataram que faziam consultas compartilhadas e que, em uma visita domiciliar, depois de identificar a necessidade de encaminhamento, o caso era partilhado com outros profissionais, o que se configurava como interdisciplinaridade, por meio da troca de saberes e da integração de conhecimentos.

Os achados da observação em relação à dimensão 'atividades individuais' divergiram dos achados dos questionários, nos quais esta dimensão apresentou de média a forte tendência interdisciplinar. Verificou-se que, na prática clínica, o trabalho era uniprofissional e com escassos momentos de integração entre os profissionais. A perspectiva interdisciplinar faz-se necessária mesmo nos atendimentos individualizados.

A concepção ampliada de saúde, que orienta o cuidado integral, implica que em todas as atividades é preciso haver integração de saberes, inclusive no atendimento individual.

No tocante à dimensão 'comunicação' (Tabela 4), os profissionais da amostra relataram que estabeleciam comunicação com outros profissionais quando verificavam a necessidade da equipe ou o apoio da rede. Esperidião, Cruz e Silva (2011) concluíram em estudo que é necessária uma parceria entre a atenção básica e a rede especializada para possibilitar o diálogo entre os profissionais dos distintos níveis de atenção e garantir a qualidade dos serviços prestados. Apesar da necessidade de que haja uma relação entre os serviços, os vínculos entre a atenção básica e a rede especializada são deficitários. O diálogo e a sistematização dessa comunicação representam uma barreira para as parcerias que ultrapassem a lógica do encaminhamento e apontem na direção da interdisciplinaridade. 
Os profissionais pesquisados afirmaram que usavam a comunicação visando à interdisciplinaridade. Um dos primeiros passos para se estabelecer o trabalho em equipe é o acordo sobre os objetivos em comum, o diálogo e a troca de informações, requisitos da dimensão 'comunicação' que facilitam a cooperação necessária para a intervenção interprofissional. O diálogo auxilia os profissionais a resolverem habilmente os problemas rotineiros que surgem em seu ambiente de trabalho, na perspectiva de melhorar a convivência entre eles e, consequentemente, a qualidade do serviço ofertado à população (Morschel e Barros, 2014).

Com a pesquisa in locus, verificou-se o quanto os aspectos subjetivos perpassam as categorias e funcionam como obstáculos para a integração de disciplinas e de profissionais, a citar: o reduzido tempo para diálogo, a criação do vínculo e a dificuldade de saber ouvir e falar com o colega, para quebrar a lógica da comunicação, que foi apontada como prevalente no dia a dia dos profissionais que responderam ao questionário quantitativo. A prática colaborativa requer intensa comunicação e interação entre os profissionais (Peduzzi et al., 2013). Quando essa comunicação não ocorre, há prejuízo para o potencial resolutivo da colaboração para enfrentamento das necessidades de saúde.

Nas quatro equipes de saúde da família, observaram-se comportamentos diferentes no que tangia à dimensão comunicação. Na equipe Rosa, o fluxo de informações era unidirecional, o diálogo inexistia pela dificuldade de relações entre os profissionais e as decisões em relação ao processo de trabalho ficavam centralizadas na figura da profissional enfermeira, distanciando-se da lógica de trabalho interprofissional. De modo diferente, na equipe Verde existia troca de informações, uma interdependência dos profissionais presenciada pelos seus momentos de conversa em que surgiam sugestões e auxílio com o trabalho do outro, e a comunicação era horizontalizada. Na equipe Azul, as atividades eram realizadas de forma descentralizada, com troca de informações e divisão das responsabilidades. Na equipe Laranja, o diálogo era pouco frequente, mas havia momentos de troca, interação e participação conjunta dos envolvidos. Evidenciou-se, nessas equipes, sobretudo na equipe Verde, importante potencial para o trabalho interprofissional e colaborativo.

Existem mecanismos que determinam como a prática colaborativa é implementada e executada, dentre os quais se destacam os relacionados ao apoio institucional, a exemplo dos modelos de governança, protocolos estruturados, recursos operacionais compartilhados, políticas de pessoal, práticas gerenciais de apoio (World Health Organization, 2010). Nessa perspectiva, considerando-se o fato de que o trabalho colaborativo, além de comunicação, necessita de coordenação das atividades, salienta-se a importância dessa dimensão institucional. As diferenças identificadas em termos de comunicação e do potencial colaborativo entre as equipes de uma mesma unidade de saúde da família apontaram para uma insuficiência no quesito coordenação, ficando a critério de cada equipe construir práticas colaborativas sem que houvesse uma 
coordenação capaz de orientá-las. Nessa perspectiva, evidenciavam falhas nos mecanismos institucionais de apoio às práticas colaborativas.

O estudo demonstrou que as dimensões dependentes da postura do profissional de forma isolada (Tabelas 3 e 4) e que não envolviam interações com o colega (atividades individuais, comunicação e atitude) foram as que tiveram as maiores médias, consideradas pelos profissionais como práticas fortemente exercidas no dia a dia do seu trabalho. Esse achado denunciou a tendência de trabalho uniprofissional nessas equipes.

O trabalho em equipe na perspectiva interprofissional possibilita otimizar as habilidades de seus membros, compartilhar o gerenciamento de casos e prestar serviços de saúde de melhor qualidade aos usuários e à comunidade. Outros mecanismos também necessários a essa perspectiva dizem respeito à cultura de trabalho, tais como: estratégias de comunicação, políticas para resolução de conflitos, processos para tomada de decisões compartilhada (World Health Organization, 2010). Todavia, esses mecanismos demandam momentos e espaços de encontro para se efetivarem, o que não se concretiza quando os profissionais não dispõem de tempo para que isso ocorra e valorizam excessivamente a atuação uniprofissional.

Segundo Pombo (2008), a interdisciplinaridade demanda não só a convergência de pontos de vista, que atinge as camadas mais profundas da realidade cognoscível, como também uma aproximação pensada do lado do sujeito e do próprio objeto de investigação e sua complexidade. Ela é útil e essencial em

Tabela 4

Escore das dimensões comunicação e atitude estratificadas por categoria profissional

\begin{tabular}{|c|c|c|c|c|}
\hline Dimensão & Amostra & Enfermeiros & Médicos & Cirurgiões-dentistas \\
\hline Comunicação & 8,60 & 8,58 & 8,64 & 8,57 \\
\hline $\begin{array}{l}\text { Durante o planejamento, as atividades } \\
\text { do serviço são discutidas de maneira } \\
\text { horizontalizada pela equipe de saúde da } \\
\text { família. }\end{array}$ & 8,13 & 8,25 & 8,08 & 8,04 \\
\hline A troca de conhecimento e o diálogo entre & & & & \\
\hline $\begin{array}{l}\text { os profissionais são realizados pela equipe } \\
\text { de saúde da família. }\end{array}$ & 8,53 & 8,48 & 8,69 & 8,44 \\
\hline $\begin{array}{l}\text { É fácil compreender a linguagem empregada } \\
\text { pelos profissionais da equipe. }\end{array}$ & 9,14 & 9,02 & 9,15 & 9,25 \\
\hline Atitude & 9,64 & 9,64 & 9,57 & 9,70 \\
\hline Respeito a crenças e valores das pessoas & & & & \\
\hline $\begin{array}{l}\text { (colegas de trabalho e usuários), bem como } \\
\text { às demais expressões da sua cultura. }\end{array}$ & 9,70 & 9,69 & 9,58 & 9,83 \\
\hline Reconheço minhas limitações e entendo que & & & & \\
\hline $\begin{array}{l}\text { posso ser ajudado pelos colegas da equipe } \\
\text { (considerar todos os membros da equipe). }\end{array}$ & 9,58 & 9,60 & 9,57 & 9,58 \\
\hline
\end{tabular}


um local de trabalho onde os profissionais devem atuar de maneira humanizada, crítica e reflexiva, com o objetivo de melhorar a qualidade dos serviços ofertados aos usuários do sistema de saúde.

Muitos profissionais de saúde já atuam em equipes e se comunicam ativamente com os colegas, mas isso não implica que estejam trabalhando na perspectiva interprofissional. Esta perspectiva pressupõe o envolvimento de uma força de trabalho de saúde colaborativa, preparada para a prática, pronta para enfrentar problemas complexos ou emergenciais e resolvê-los em conjunto (World Health Organization, 2010). Tal posicionamento requer apoio institucional, traduzido em iniciativas da gestão para implementar o trabalho colaborativo, mas exige também um comprometimento pessoal, uma vez que a interprofissionalidade requer uma articulação intencional entre as profissões (Costa et al., 2015). A falta dessa intencionalidade, que está relacionada à dimensão subjetiva da prática profissional, dificulta a colaboração.

\section{Considerações finais}

A confrontação entre os achados dos questionários e da observação participante revelou contradições importantes. Ao se analisarem isoladamente os dados quantitativos, verificou-se uma aproximação das equipes com a perspectiva interdisciplinar e interprofisssional do trabalho em saúde. Entretanto, na observação participante foram identificadas diversas fragilidades no cotidiano de trabalho desses profissionais, parecendo evidenciar que, embora os profissionais sinalizassem uma perspectiva interdisciplinar no trabalho realizado, não o conduziam na perspectiva interprofissional.

$\mathrm{Na}$ realidade das equipes observadas, e considerando-se os resultados dos questionários, constatou-se a presença de numerosas barreiras ou limites para a interprofissionalidade, que apontaram o dissenso entre o declarado e o observado. Tal observação pode representar que embora os trabalhadores defendam a concepção interdisciplinar, têm dificuldade em empregá-la no cotidiano de trabalho, de modo que ela não se traduz majoritariamente em trabalho interprofissional e colaborativo.

A equipe multidisciplinar precisa conduzir suas práticas para reflexões conjuntas, em que a colaboração da equipe possa reconhecer as lacunas na perspectiva do trabalho interprofissional e que, efetivamente, haja uma interpenetração das 'disciplinas' e integração entre as práticas profissionais, auxiliando o processo de construção da interprofissionalidade na saúde.

A superação dos limites impostos à concretização da interdisciplinaridade por meio da intervenção interprofissional implica o enfrentamento de alguns desafios. Um deles diz respeito à lógica da profissionalização, tornando necessário encarar o antagonismo entre o modelo da lógica profissional e o modelo de colaboração interprofissional (Furtado, 2009). Nesse sentido, destaca-se a 
educação permanente voltada para a interprofissionalidade, como importante estratégia para problematizar essa questão e construir soluções possíveis para superar as dificuldades de concretização dessa intervenção. Outra perspectiva importante é a integração com processos formativos pautados na lógica da interprofissionalidade, a exemplo das residências multiprofissionais.

Faz-se necessário, também, readequar o modelo formativo vigente nos cursos de graduação em saúde. A educação interprofissional vem se apresentando como importante estratégia para a construção do trabalho colaborativo e interprofissional, conforme apontam diversos estudos (Barr e Low, 2013; Costa, 2016; Reeves, 2016). Essa tendência pode contribuir, a posteriori, para que os profissionais assumam uma relação de diálogo com sua equipe, partilhem saberes, façam trocas e parcerias e promovam integração e responsabilização, numa perspectiva interdisciplinar sobre a condução de cuidado em saúde integral. Para ultrapassar os obstáculos impostos nos dias atuais, é imprescindível que o diálogo aponte as evidências científicas de prognóstico advindo de ações interdisciplinares, a fim de sensibilizar os profissionais para o trabalho interdisciplinar e interprofissional.

Outro obstáculo a ser superado relaciona-se à sobrecarga de trabalho dessas equipes e à falta de tempo disponível para a integração entre os profissionais. Destaca-se, nesse sentido, a necessidade de mecanismos institucionais, traduzidos em ações em nível da gestão que possibilitem essas práticas e favoreçam o investimento subjetivo dos trabalhadores na realização delas.

É importante, ainda, que os resultados encontrados no estudo apresentado neste artigo subsidiem discussões e contribuam para mudanças no trabalho das equipes de saúde da família, de modo a contribuir para o incentivo à efetivação da saúde com qualidade e resolubilidade, ao tornar os profissionais aptos a atuarem na direção da interdisciplinaridade, visando à colaboração e ao trabalho interprofissional.

\section{Colaboradores}

Danyelle Nóbrega de Farias conduziu a pesquisa de campo e atuou em todas as etapas, da redação do projeto até a construção do artigo, com base em sua dissertação de mestrado. Kátia Suely Queiroz Silva Ribeiro e Ulisses Umbelino dos Anjos orientaram a dissertação de mestrado e auxiliaram na redação e na revisão crítica do artigo. Geraldo Eduardo Guedes de Brito participou de todas as etapas desde o planejamento, desenvolvimento e revisão do artigo. Não há conflito de interesses. 
Resumen La interdisciplinariedad y su correlación práctica, la interprofesionalidad, son esenciales para el trabajo en la Estrategia Salud de la Familia. Se objetivó caracterizar la perspectiva interdisciplinaria en la Estrategia Salud de la Familia en una capital de Brasil relacionándola con la interprofesionalidad. Se realizó un estudio transversal, exploratorio y descriptivo, con los abordajes cualitativo y cuantitativo. Los datos fueron recolectados en el periodo de junio a noviembre de 2014 por medio de cuestionarios y observación participante. Los resultados del cuestionario se convirtieron en puntuaciones, permitiendo clasificar el nivel de interdisciplinariedad. Los hallazgos de la observación, registrados en el diario de campo, fueron sometidos al análisis de contenido y discutidos en paralelo con los hallazgos cuantitativos. Se constató, en los cuestionarios, que la dimensión 'acogida' fue la única que presentó media disponibilidad para prácticas que pueden viabilizar la interdisciplinariedad. Los resultados apuntan que la percepción de los profesionales de salud acerca de la interdisciplinariedad fue positiva, pero se verificaron contradicciones de estos hallazgos con el observado in locus. En la observación participante, se constató que los profesionales necesitan recorrer algunos caminos en el sentido de materializar la interdisciplinariedad en prácticas interprofesionales colaborativas. Se destaca la necesidad de acciones a nivel de la gestión que favorezcan esas prácticas; la educación permanente como estrategia de enfrentamiento de las dificultades de integración; y la inversión subjetiva de los trabajadores en la misma dirección.

Palabras clave comunicación interdisciplinaria; investigación interdisciplinaria; equipo de salud interdisciplinario; relaciones interprofesionales.

\section{Notas}

${ }^{1}$ Universidade Federal da Paraíba, Departamento de Estatística, Programa de Pós-Graduação em Modelos de Decisão e Saúde, João Pessoa, Paraíba, Brasil. < danynobregadefarias@ hotmail.com>

Correspondência: Universidade Federal da Paraíba, s/n, CEP 58059-900, Centro de Ciências da Saúde, Campus I, Departamento de Fisioterapia, Cidade Universitária, João Pessoa, Paraíba, Brasil.

${ }^{2}$ Universidade Federal da Paraíba, Departamento de Fisioterapia, João Pessoa, Paraíba, Brasil. $<$ katiaqsribeiro@yahoo.com.br>

${ }^{3}$ Universidade Federal da Paraíba, Departamento de Estatística, Programa de Pós-Graduação em Modelos de Decisão e Saúde, João Pessoa, Paraíba, Brasil.

$<$ ulisses@de.ufpb.br>

${ }^{4}$ Universidade Federal da Paraíba, Departamento de Fisioterapia, João Pessoa, Paraíba, Brasil. $<$ eduardo.guedes.ufpb@gmail.com>

${ }^{5}$ A pesquisa foi resultado da dissertação de mestrado $A$ interdisciplinaridade na prática profissional na Estratégia Saúde da Família (Farias, 2014). 


\section{Referências}

BARDIN, Laurence. Análise de conteúdo. Lisboa: Edições 70, 2011.

BARR, Hugh et al. Effective Interprofessional Education: argument, assumption and evidence. Oxford: Blackwell, 2005.

BARR, Hugh; LOW, H. Introducing Interprofessional Education. Fareham: CAIPE, 2013.

BATISTA, Karina B. C.; GONÇALVES, Otília S. J. Formação dos profissionais de saúde para o SUS: significado e cuidado. Saúde e Sociedade, São Paulo, v. 20, n. 4, p. 884-899, 2011. Disponível em: <http://dx.doi.org/10.1590/S0104$12902011000400007>$. Acesso em: 27 jan. 2016.

BRASIL. Ministério da Saúde. Secretaria de Atenção à Saúde. Departamento de Atenção Básica. Diretrizes do Nasf: Núcleo de Apoio à Saúde da Família. Brasília: Ministério da Saúde, 2009 (Cadernos de Atenção Básica, 27). Disponível em: <https://www.nescon.medicina.ufmg.br/ biblioteca/imagem/caderno_atencao_basica_ diretrizes_nasf.pdf>. Acesso em: 27 jan. 2016.

BRASIL. Ministério da Saúde. Política Nacional de Humanização (PNH): HumanizaSUS - documento-base. 4. ed. Brasília: Ministério da Saúde, 2010. Disponível em: <http://bibliotecadigital. puc-campinas.edu.br/services/e-books/humanizasus_documento_gestores_trabalhadores_sus. pdf > . Acesso em: 27 jan. 2016.

BRASIL. Ministério da Saúde. Secretaria de Atenção à Saúde. Departamento de Atenção Básica. Política Nacional de Atenção Básica (PNAB). Brasília: Ministério da Saúde, 2012. Disponível em: <http://189.28.128.100/dab/docs/publicacoes/geral/pnab.pdf> . Acesso em: 7 dez. 2016.

BUSSAB, Wilton O.; MIAZAKI, Édina S.; ANDRADE, Dalton F. Introdução à análise de agrupamentos. In: SIMPÓSIO NACIONAL DE PROBABILIDADE E ESTATÍSTICA, 9., São Paulo, 1990. Anais. São Paulo: ABE, 1990. Disponível em: <https://pt.scribd.com/ doc/4567031/Livro-de-Analise-de-Agrupamento >. Acesso em: 27 jan. 2016.
CLEMENTINO, Francisco S. et al. Acolhimento na atenção básica: análise a partir da avaliação externa do Programa de Melhoria do Acesso e da Qualidade da Atenção Básica (PMAQ-AB). Revista Saúde e Ciência Online, Campina Grande, v. 4, n. 1, p. 62-80, 2015. Disponível em: <http://www.ufcg.edu.br/revistasaudeeciencia/index.php/RSC-UFCG/article/ viewFile/229/160>. Acesso em: 27 jan. 2016.

COSTA, Glauce D. et al. Saúde da família: desafios no processo de reorientação do modelo assistencial. Revista Brasileira de Enfermagem, Brasília, v. 62, n. 1, p. 113-118, 2009. Disponível em: <http://dx.doi.org/10.1590/ S0034-71672009000100017>. Acesso em: 27 jan. 2016.

COSTA, Marcelo V. The interprofessional education in Brazilian context: some reflections. Interface: Comunicação, Saúde e Educação, Botucatu, v. 20, n. 56, p. 197-198, 2016. Disponível em: <http:// www.redalyc.org/pdf/1801/180142937017.pdf>. Acesso em: 8 dez. 2016.

COSTA, Marcelo V. et al. Pró-Saúde e PET-Saúde como espaços de educação interprofissional. Interface: Comunicação, Saúde e Educação, Botucatu, v. 19, supl. 1, p. 709-720, 2015. Disponível em: <http://dx.doi.org/10.1590/180757622014.0994>. Acesso em: 6 dez. 2016.

DE BRITTO JÚNIOR, Álvaro F.; FERES JÚNIOR, Nazir. A utilização da técnica da entrevista em trabalhos científicos. Evidência: Olhares e Pesquisa em Saberes Educacionais, Araxá, v. 7, n. 7, p. 237-250, 2011. Disponível em: $<$ http://www.uniaraxa.edu.br/ojs/index.php/ evidencia/article/view/200/186>. Acesso em: 29 jan. 2016.

ESPERIDIÃO, Elizabeth; CRUZ, Maryana F. R.; SILVA, Gelda A. Perfil e atuação dos enfermeiros da rede especializada em saúde mental de Goiânia-GO. Revista Eletrônica de Enfermagem, Brasília, v. 13, n. 3, p. 493-501, 2011. Disponível em: <https://www.fen.ufg. br/fen_revista/v13/n3/pdf/v13n3a15.pdf>. Acesso em: 28 jan. 2016. 
FARIAS, Danyelle N. A interdisciplinaridade na prática profissional na Estratégia da Saúde da Família. 92 f. Dissertação (Mestrado em Modelos de Decisão e Saúde) - Programa de Pós-Graduação em Modelos de Decisão e Saúde, Universidade Federal da Paraíba, João Pessoa,Paraíba, 2014.

FEUERWERKER, Laura C. M.; CECÍLIO, Luiz C. O. O hospital e a formação em saúde: desafios atuais. Ciência \& Saúde Coletiva, Rio de Janeiro, v. 12, n. 4, p. 965-971, 2007. Disponível em: <http://dx.doi.org/10.1590/S1413$81232007000400018>$. Acesso em: 27 jan. 2016.

FURTADO, Juarez P. Arranjos institucionais e gestão da clínica: princípios da interdisciplinaridade e interprofissionalidade. Cadernos Brasileiros de Saúde Mental, Florianópolis, v. 1, n. 1, p. 1-11, 2009. Disponível em: <http://incubadora.periodicos. ufsc.br/index.php/cbsm/article/view/1013/1136>. Acesso em: 19 maio 2017.

GARUZI, Miriane et al. Acolhimento na Estratégia Saúde da Família: revisão integrativa. Revista Panamericana de Salud Publica, Washington, v. 35, n. 2, p. 144-149, 2014. Disponível em: <http://www.scielosp.org/pdf/rpsp/v35n2/ a09v35n2.pdf $>$. Acesso em: 2 fev. 2016.

JAPIASSU, Hilton. Interdisciplinaridade $e$ patologia do saber. Rio de Janeiro: Imago, 1976.

MACHADO, Maria F. A. S et al. Integralidade, formação de saúde, educação em saúde e as propostas do SUS: uma revisão conceitual. Ciência \& Saúde Coletiva, Rio de Janeiro, v. 12, n. 2, p. 335-342, 2007. Disponível em: <http:// dx.doi.org/10.1590/S1413-81232007000200009>. Acesso em: 27 jan. 2016.

MALTA, Débora C.; MERHY, Emerson E. O percurso da linha do cuidado sob a perspectiva das doenças crônicas não transmissíveis. Interface: Comunicação, Saúde e Educação, Botucatu, v. 14, n. 34, p. 593-606, 2010. Disponível em: <http://www.scielo.br/pdf/icse/v14n34/ aop0510.pdf $>$. Acesso em: 27 jan. 2016.

MORSCHEL, Aline; BARROS, Maria E. B. Processos de trabalho na saúde pública: humanização e efetivação do Sistema Único de Saúde. Revista Saúde e Sociedade, São Paulo, v. 23, n. 3, p. 928-941, 2014. Disponível em: <http://dx. doi.org/10.1590/S0104-12902014000300016>. Acesso em: 28 jan. 2016.

OLIVEIRA, Hadelânia M.; PIRES, Thiago O.; PARENTE, Rosana C. P. As relações de poder na Estratégia Saúde da Família sob o enfoque da teoria de Hannah Arendt. Saúde \& Transformação Social, Santa Catarina, v. 1, n. 2, p. 17-26, 2011. Disponível em: <http:// incubadora.periodicos.ufsc.br/index.php/ saudeetransformacao/article/view/494/0>. Acesso em: 2 fev. 2016.

PEDUZZI, Marina et al. Educação interprofissional: formação de profissionais de saúde para o trabalho em equipe com foco nos usuários. Revista da Escola de Enfermagem da USP, São Paulo, v. 47, n. 4, p. 977-983, 2013. Disponível em: <http:// dx.doi.org/10.1590/S0080-623420130000400029>. Acesso em: 6 dez. 2016.

PIRES, Maria R. G. M.; GÖTTEMS, Leila B. D. Análise da gestão do cuidado no Programa de Saúde da Família: referencial teórico-metodológico. Revista Brasileira de Enfermagem, Brasília, v. 62, n. 2, p. 294-299, 2010. Disponível em: <http://dx.doi.org/10.1590/S003471672009000200020 > . Acesso em: 29 jan. 2016.

POMBO, Olga. Epistemologia da interdisciplinaridade. Revista do Centro de Educação e Letras da Unioeste, Foz do Iguaçu, v. 10, n. 1, p. 9-40, 2008.

REEVES, Scott. Porque precisamos da educação interprofissional para um cuidado efetivo e seguro. Interface: Comunicação, Saúde e Educação, Botucatu, v. 20, n. 56, p. 185-196, 2016. Disponível em: <http://www.scielosp.org/pdf/ icse/v20n56/pt_1807-5762-icse-20-56-0185. pdf>. Acesso em: 8 dez. 2016.

SAUPE, Rosita et al. Competência dos profissionais da saúde para o trabalho interdisciplinar. Interface: Comunicação, Saúde e Educação, Botucatu, v. 9, n. 18, p. 521-536, 2005. Disponível em: <http://www.scielosp.org/pdf/icse/v9n18/ a05v9n18.pdf>. Acesso em: 8 fev. 2016. 
WORLD HEALTH ORGANIZATION (WHO).

Framework for action on interprofessional education and collaborative practice. Geneva: WHO, 2010.

ZANETTI, Tatiele G. et al. Perfil socioprofissional e formação de profissionais de equipes de saúde da família: um estudo de caso. Ciência, Cuidado e Saúde, Maringá, v. 9, n. 3, p. 448-455, 2010.

Recebido em 26/04/2016.

Aprovado em 24/05/2017. 
